\title{
Submassive Pulmonary Embolism: Risk Evaluation and Role of Fibrinolysis
}

\author{
Rathasen Prom • Ryan Dull - Stephanie Z. Kujawski • \\ Iwona Rybak · John Fanikos
}

Published online: 28 September 2013

(C) Springer Science+Business Media New York 2013

\begin{abstract}
Submassive pulmonary embolism (PE) is characterized by hemodynamic stability with evidence of right ventricular dysfunction or myocardial necrosis, and represents a heterogeneous population at risk for adverse outcomes. Although patients with this subtype of PE are at higher risk of death, it is unclear whether escalation of care with fibrinolytic therapy mitigates this risk. The controversial role of fibrinolytic therapy for submassive PE is driven by a paucity of data, conflicting results from clinical trials, and lack of reliable positive predictive markers of mortality in this population. When compared with anticoagulation therapy alone, systemic fibrinolytic therapy leads to a more rapid improvement in pulmonary artery hemodynamics, restoration of right ventricular function, improved lung perfusion, and fewer episodes of clinical deterioration. The clinical significance of these findings
\end{abstract}

R. Prom ( $\square)$

Department of Pharmacy, Mission Hospitals,

509 Biltmore Avenue, Asheville, NC 28801, USA

e-mail: rathasen.prom@gmail.com

R. Dull

Department of Pharmacy Practice, Creighton University,

2500 California Plaza, Omaha, NE 68178, USA

S. Z. Kujawski

UNC Eshelman School of Pharmacy, Mission Hospitals,

509 Biltmore Avenue, Asheville, NC 28801, USA

I. Rybak · J. Fanikos

Department of Pharmacy, Brigham and Women's Hospital,

75 Francis Street, Boston, MA 02115, USA remains uncertain because fibrinolytic therapy has not been demonstrated to improve mortality or reduce recurrent PE in patients with submassive PE. However, it is unclear whether this reflects methodological limitations such as small sample size and rescue fibrinolytic therapy in those assigned placebo, the absence of benefit in all patients with submassive PE, or the benefit is limited to an undefined, high-risk subset. The decision to administer adjunctive fibrinolytics in patients with submassive PE should be made on an individual basis, with serious consideration given to those with severe PE-related clinical manifestations and an acceptable risk of bleeding.

Keywords Thrombolytics · Fibrinolytics · Pulmonary embolism $\cdot$ Submassive $\cdot$ Massive

\section{Introduction}

In the US, venous thromboembolic (VTE) disease affects an estimated 300,000-600,000 individuals each year, and approximately one-third of patients seeking medical care for VTE are diagnosed with pulmonary embolism (PE) [1]. The prognosis and outcomes associated with $\mathrm{PE}$ are diverse. Patients with PE are at risk for morbid outcomes such as recurrent VTE and chronic thromboembolic pulmonary hypertension (CTEPH); moreover, PE is the primary cause of mortality associated with VTE, the leading cause of preventable hospital death, and a common cause of sudden death [1]. Acute PE has a correspondingly wide range of clinical manifestations, many of which are nonspecific and sometimes asymptomatic, making a rapid diagnosis difficult to establish. Despite advances in the tools used to identify PE, it is often undetected and continues to be a challenging diagnosis [2]. 
Therapeutic anticoagulation reduces the incidence of recurrent VTE by inhibiting the growth and propagation of formed thrombi. It is a proven treatment modality recommended for all patients with highly suspected or confirmed

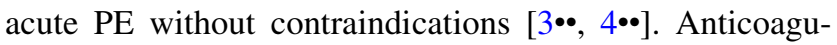
lation therapy does not, however, accelerate lysis of existing thrombi and is not effective for prompt restoration of perfusion in patients with cardiovascular collapse; the adjunctive use of fibrinolytics would be needed to yield these effects. When compared with anticoagulation therapy alone, systemic fibrinolytic therapy leads to a more rapid improvement in pulmonary artery hemodynamics, restoration of right ventricular (RV) function, and improved lung perfusion $[3 \bullet \bullet, 4 \bullet \cdot]$. However, fibrinolytic therapy has not been demonstrated to improve mortality or prevent recurrent VTE in non-massive PE and is associated with a higher risk of serious bleeding [3••, $4 \bullet \bullet]$. Therefore, the net clinical benefit of fibrinolytic therapy in patients with acute PE appears to have the greatest potential when it is given early to patients with clinical characteristics associated with a high risk of death.

The considerable risk of early mortality as well as the serious adverse effects associated with treatment of acute PE highlights the need for prompt risk stratification to identify candidates who may require escalation of care, including fibrinolytic therapy. Outcomes from multicenter, international registry data derived from patients with acute PE consistently identified sustained hypotension and circulatory arrest as indicators of substantially higher mortality [5, 6]. Accordingly, massive $\mathrm{PE}$ is defined as sustained hypotension, pulselessness, or persistent brady-

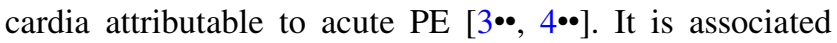
with a high mortality rate but accounts for fewer than $5 \%$ of all patients with acute PE [4••, 5]. Patients with massive $\mathrm{PE}$ are widely accepted as eligible for more aggressive interventions such as systemic or catheter-directed fibrinolytic therapy or surgical embolectomy $[3 \bullet \bullet, 4 \bullet \bullet]$.

In contrast with massive $\mathrm{PE}$, patients with neither hypotension nor evidence of RV dysfunction (RVD) account for the majority of patients with acute PE, have a more favorable prognosis, and are considered low risk for adverse outcomes when treated with anticoagulation ther-

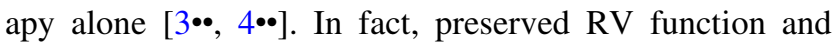
normal cardiac biomarkers portend excellent negative predictive value and may be useful for identifying patients suitable for early discharge and outpatient therapy [7]. Among patients with acute PE, this subtype is the least likely to benefit from fibrinolytic therapy.

Submassive PE is characterized by the presence of hemodynamic stability with evidence of RVD or myocardial necrosis and represents a heterogeneous population that accounts for approximately $20-25 \%$ of patients with acute PE [4••, 8]. However, unlike massive and low-risk PE subtypes, fibrinolytic therapy for submassive PE is an area of significant therapeutic controversy complicated by a paucity of data and difficulty in early recognition of patients most likely to benefit. The purpose of this article is to evaluate the risks and review the fibrinolysis-related clinical trial outcomes associated with submassive PE.

\section{Submassive Pulmonary Embolism: Definition and Risk Evaluation}

In normotensive patients with acute PE, evidence of RVD or myocardial necrosis is associated with heightened risk for early mortality and adverse outcomes; these patients are considered to have submassive PE. Several common diagnostic tools (electrocardiography, echocardiography, computed tomography) and laboratory tests [brain natriuretic peptide (BNP), $N$-terminal pro-BNP (NT-proBNP)] can be employed to determine if RVD exists, and troponin levels can be obtained to assess for myocardial necrosis.

\section{Right Ventricular Dysfunction}

In a prospective study conducted in hemodynamically stable PE patients, $9.2 \%$ of those with RVD developed PE-related shock and $4.6 \%$ died; in contrast, these complications were absent in patients without RVD [9]. The available data, which include the MAPPET-3 and ICOPER registries, prospective studies, and meta-analyses, have related echocardiographic- or computed tomography-determined RVD with a 2- to 2.57-fold excess risk of mortality within 30 days and a high negative predictive value approaching $100 \%$ for ruling out an adverse outcome in normotensive PE patients [1014]. However, use of RVD alone has been shown to be insufficient for predicting PE-related mortality (positive predictive value of $\sim 5-12 \%$ ) and the composite of PErelated death or clinical deterioration [12, 14-16].

Elevated levels of BNP or NT-proBNP are significantly associated with RVD [7, 17]. In a systematic review of hemodynamically stable PE patients, high BNP and NTproBNP concentrations corresponded with a 9.5- and 5.7fold increased risk of in-hospital or 30-day mortality, respectively [13]. Although both markers were modestly accurate at ruling out an adverse outcome (BNP's negative predictive value $=76 \%$; NT-proBNP's negative predictive value $=81 \%$ ), they were not reliable for predicting inhospital or 30-day survival or the composite of in-hospital PE-related death or clinical deterioration [13, 15].

\section{Myocardial Necrosis}

In a study of normotensive patients with acute PE, all inhospital deaths $(12.5 \%)$ occurred in those with elevated 
troponin $\mathrm{T}$ levels, among which the in-hospital mortality rate was $25 \%$ [18]. Results of prospective studies and meta-analyses performed in hemodynamically stable PE patients have correlated troponin I or T elevation with a 2.66- to 21-fold increased risk of PE-related mortality and 4.12- to 24.1-fold excess risk of adverse clinical events [13, $14,18,19]$. The use of these cardiac biomarkers may be helpful for ruling out (negative predictive value $95.8 \%$ ) the development of PE-related death; however, they are unreliable for ruling it in (positive predictive value $10.5 \%$ ) [14]. In a multicenter, prospective study by Bova et al., troponin I was not a reliable predictor of the composite of in-hospital PE-related death or clinical deterioration [15].

Right Ventricular Dysfunction Combined with Myocardial Necrosis

The presence of both RVD and elevated troponin has been associated with a 5.6-fold increased risk of 30-day mortality in normotensive PE patients [20]. Their combined use, however, is still inadequate (negative predictive $94.7 \%$; positive predictive value $15.2 \%$ ) for predicting PE-related mortality [14].

The American College of Chest Physicians (ACCP) and American Heart Association (AHA) recommend assessment of hemodynamics and RV function to distinguish between massive, submassive, and low-risk subtypes (Table 1) $[4 \cdot \bullet]$.

Table 1 Acute pulmonary embolism subgroup definitions [4••]

\begin{tabular}{ll}
\hline Subgroup & Definition \\
\hline Massive & Sustained hypotension not due to a cause other than PE \\
& SBP $<90 \mathrm{mmHg}$ for at least $15 \mathrm{~min}$ or requiring \\
inotropic support & Persistent profound bradycardia \\
& HR $<40$ bpm with signs or symptoms of shock \\
& Pulselessness \\
RVD without hypotension \\
RV dilation (LV:RV diameter $>0.9$ on computed \\
tomography or echocardiography) \\
RV systolic dysfunction on echocardiography \\
Elevation of BNP ( $>90$ pg/ml) \\
Elevation of NT-proBNP $(>500 \mathrm{pg} / \mathrm{ml})$ \\
Electrocardiographic changes \\
Myocardial necrosis without hypotension \\
Elevation of troponin I $(>0.04 \mathrm{ng} / \mathrm{ml})$ \\
Elevation of troponin T ( $>0.1 \mathrm{ng} / \mathrm{ml})$ \\
No evidence of RVD, myocardial necrosis, or \\
hypotension
\end{tabular}

$\overline{B N P}$ brain natriuretic peptide, $H R$ heart rate, $L V$ left ventricle, $N T$ proBNP N-terminal pro-BNP, $P E$ pulmonary embolism, $R V$ right ventricle, $R V D$ right ventricular dysfunction, $S B P$ systolic blood pressure

\section{Fibrinolytics: Mechanism of Action}

Fibrinolytic agents work by enhancing the conversion of inactive plasminogen within the circulation to active plasmin [21]. Both circulating and fibrin-bound plasminogen have the potential to be converted to the protease plasmin depending on the fibrin specificity of the fibrinolytic used. Once generated, plasmin acts on fibrin clots by cleaving both fibrinogen and fibrin crosslinks [22]. Degradation of coagulation factors $\mathrm{V}$ and VIII may also be possible with nonspecific plasmin. These processes of plasminogen activation and fibrinolysis continue until plasminogen activator inhibitor type-1 (PAI-1) or alpha-2 antiplasmin form a stable complex with free plasmin, which results in inactivation of this enzyme and cessation of the degradation phase. The older generation plasminogen activators such as streptokinase and urokinase are classified as nonspecific fibrinolytics due to their ability to lyse thrombus without fibrin specificity [23, 24]. The newer generation fibrinolytics, which include alteplase, reteplase, and tenecteplase, are classified as fibrin-specific and are more efficient at dissolving thrombi without depleting systemic coagulation factors, in contrast to non-fibrin specific agents [22, 25-27]. Dissolution of physiologic thrombi in addition to enhanced systemic activity of the nonspecific plasmin contributes to the bleeding complications associated with administration of fibrinolytics. Furthermore, agents that do not induce a significant depletion in circulating alpha-2 antiplasmin demonstrate less of a systemic fibrinolytic effect [27]. Pharmacokinetic and pharmacodynamic properties of fibrinolytics are summarized in Table 2.

\section{Efficacy Outcomes Associated with Fibrinolysis in Submassive Pulmonary Embolism}

The results of double-blind, randomized, controlled clinical trials evaluating fibrinolytics in submassive PE are presented in Table 3.

\section{Right Ventricular Function and Lung Perfusion}

The adjunctive use of fibrinolytics in normotensive patients with acute PE and RVD results in quicker resolution of RV impairment and perfusion lung defect compared to treatment with anticoagulation alone [28, 29]. Evidence from two small randomized trials [TIPES $(n=58)$, Fasullo et al. $(n=72)]$ demonstrated superior RV function improvements at $24 \mathrm{~h}(p=0.04$ and $p<0.0001)$ with fibrinolytic therapy compared to placebo [28, 29]. It is unclear, however, if this effect is maintained over time. TIPES indicated RVD reduction may not differ between 


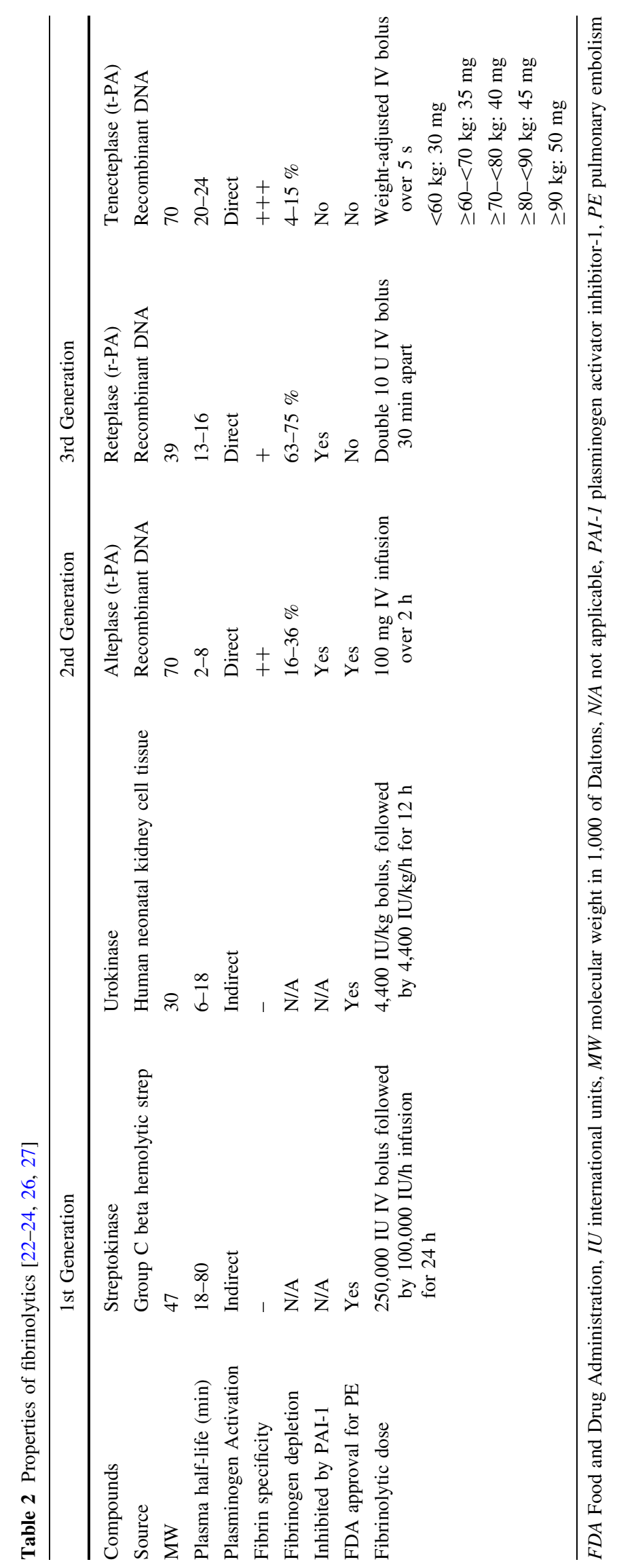




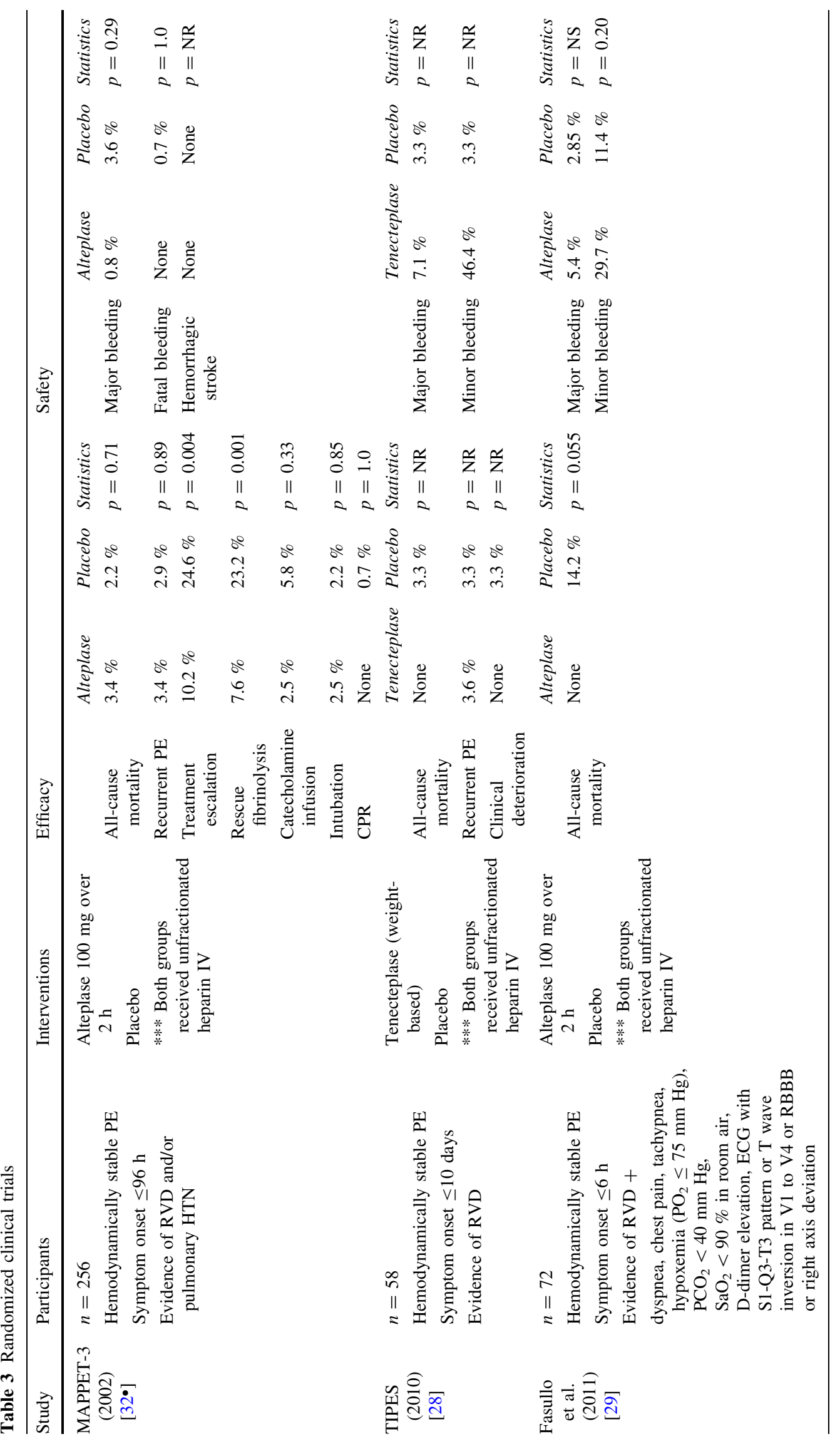


fibrinolysis and placebo at 7 days ( $p=$ not significant), while Fasullo et al. suggested the benefit may persist throughout hospitalization $(p<0.0001)$ and also at 6 months $(p<0.0001)[28,29]$. In one retrospective cohort study $(n=128)$, fibrinolytic therapy yielded a larger relative improvement in perfusion lung defect compared to heparin monotherapy at 1 week (54 vs. $42 \%$; $p=0.01$ ); additionally, a greater proportion of fibrinolysistreated individuals attained $>50 \%$ relative improvement in perfusion lung defect (57 vs. $37 \% ; p=$ not reported) [30].

Mortality

Despite more rapidly resolving RVD, use of fibrinolysis in hemodynamically stable PE patients who have RVD does not appear to be effective for improving short-term survival. The initial evidence from early non-randomized studies provided conflicting results; one retrospective investigation suggested fibrinolysis might not affect inhospital PE-related death, whereas subgroup analyses of a randomized trial and the MAPPET- 3 registry alluded to reduced 30-day mortality with fibrinolysis [10, 30, 31]. Since these publications, the findings of four randomized studies (MAPPET-3, TIPES, Fasullo et al., PEITHO) exploring the role of fibrinolytics in normotensive PE patients who have RVD have been reported. MAPPET-3, the first randomized and second largest trial to date, concluded fibrinolysis does not reduce in-hospital all-cause mortality $(p=0.71)$ in hemodynamically stable PE patients with RVD and/or pulmonary hypertension [32•]. TIPES did not show any differences or reveal any trends, while Fasullo et al. suggested fibrinolysis may favorably impact short-term survival; both studies were small, however, and not adequately powered to detect differences in clinical outcomes $[28,29]$. In the more recent PEITHO trial (not yet published), the largest study to date that included normotensive PE with RVD patients who also had evidence of myocardial necrosis, there were no differences in 7-day $(p=0.43)$ and 30-day mortality $(p=0.42)$ between those randomized to fibrinolysis and placebo [33*0]. Several factors may be predictive of mortality risk and adverse outcomes. In MAPPET- 3 , age $>70$ years, female gender, and arterial hypoxemia were each associated with a significant 2.29-, 2.68-, and 3.57-fold increased risk of in-hospital death or treatment escalation, respectively [32•].

\section{Recurrent Pulmonary Embolism}

Current evidence suggests adjunctive fibrinolysis may not be useful for preventing recurrent PE in acute PE patients who are hemodynamically stable and have RVD. While 
one subgroup analysis provided hope of a benefit and a positive trend was noted in one small randomized trial with fibrinolysis, results of other studies indicate a lack of effect [29, 31]. A retrospective cohort study and meta-analysis found comparable rates of recurrent PE between individuals receiving adjunctive fibrinolysis and heparin monotherapy [30, 34]. The MAPPET-3 and PEITHO trials confirmed these findings, with no observed differences between fibrinolysis and placebo for in-hospital $(p=0.89)$ and 7-day recurrent PE $(p=0.12)$, respectively [32•, 33••].

Hemodynamic Collapse and Escalation of Therapy

In spite of a lack of benefit on early mortality and recurrent $\mathrm{PE}$, adjunctive fibrinolysis significantly decreases the risk of clinical deterioration and the need for additional interventions in hemodynamically stable PE patients with RVD. In MAPPET-3 and PEITHO, rates of in-hospital treatment escalation [composite of catecholamine infusion, rescue fibrinolysis, endotracheal intubation, cardiopulmonary resuscitation (CPR), embolectomy, or thrombus fragmentation] and 7-day hemodynamic collapse (composite of CPR, hypotension or drop in blood pressure, or catecholamine infusion) were significantly lower in patients receiving fibrinolysis compared to placebo $(p=0.004$ and $p=0.002)$, respectively $[32 \bullet, 33 \bullet \bullet$. The use of rescue fibrinolysis was the main driver of reduced in-hospital treatment escalation with fibrinolytic therapy in MAPPET-3 $(p=0.001)$, a benefit also evident in PEITHO $(p<0.001)$ $[32 \bullet, 33 \bullet$. A review of secondary outcomes indicates fibrinolysis may not lead to reduced catecholamine initiation (MAPPET-3: $p=0.33$; PEITHO: $p=$ not reported), intubation (MAPPET-3: $p=0.85$; PEITHO: $p=0.13$ ), and CPR (MAPPET-3: $p=1.0$; PEITHO: $p=$ not reported). [32•, 33••] The benefits of fibrinolysis on clinical deterioration seen in MAPPET-3 and PEITHO may extend beyond the index hospitalization; in TIPES, more patients in the placebo group tended to be hospitalized for clinical worsening due to RVD at 6 months' follow-up (8.6 vs. $0 \%$; $p=$ not significant) [29].

\section{Quality of Life}

Use of fibrinolysis in hemodynamically stable patients with RVD may confer an improved quality of life at long-term follow-up. In an analysis of normotensive PE patients ( $n=162$ ) treated with adjunctive fibrinolysis or heparin alone, no patients treated with fibrinolytics had an RV systolic pressure (RVSP) at 6 months' follow-up that was greater than that at diagnosis [35]. In contrast, $27 \%$ of heparin-only patients had worsened RVSP at 6 months, $46 \%$ of which had dyspnea with limited activity or at rest (New York Heart Association $\geq 3$ ) or exercise intolerance (6-min walking distance $<330 \mathrm{~m}$ ) [35]. These findings suggest fibrinolysis may reduce the development of CTEPH. When the analysis was limited to those who had RVSP $>30 \mathrm{mmHg}$ at baseline, however, only $9 \%$ of heparinmonotherapy patients had an RVSP increase at 6 months [35].

\section{Complications of Fibrinolysis}

Table 3 also summarizes key safety results, including total major, intracranial, fatal, and minor bleedings, from randomized clinical trials evaluating the use of fibrinolysis in submassive PE.

The occurrence of serious bleeding complications is the main concern associated with fibrinolytic therapy. The available data, however, have not consistently demonstrated higher rates of total major or severe bleeding with adjunctive fibrinolysis in hemodynamically stable PE patients with RVD. There were more severe hemorrhagic episodes in those receiving fibrinolysis compared to heparin monotherapy in one retrospective cohort study (9.4 vs. $0 \% ; p=0.028$ ), while similar rates of major bleeding were observed between fibrinolysis and control groups in a meta-analysis (RR 0.86; CI 0.18-4.03) [30, 34]. The results of randomized trials have also been conflicting. In MAPPET-3 and Fasullo et al., there were no differences in major bleeding between fibrinolysis and placebo groups [29, 32•]. The larger PEITHO trial, in contrast, saw higher rates of non-intracranial major bleeding within 7 days in the fibrinolytic group $(p<0.001)$ [33••]. The reasons for these discordant findings are unclear, but one possible explanation could be differing patient populations. The mean age in MAPPET-3 and Fasullo et al. was 62 years and 56 years, respectively. Since patients enrolled in PEITHO were older (mean age 66 years) and $32 \%$ were age $>75$ years, a relative contraindication to use of fibrinolytics, they may have been at a higher risk of bleeding. There is currently no validated tool for predicting bleeding risk with fibrinolytics in PE [3••]. Table 4 lists contraindications and warnings regarding fibrinolytics [3••].

The administration of fibrinolytics in hemodynamically stable PE patients with RVD may produce higher rates of intracranial bleeding or hemorrhagic strokes and similar rates of fatal bleeding compared to treatment with anticoagulation alone. A retrospective cohort study showed that inhospital intracranial bleeding tended to occur more commonly in those receiving adjunctive fibrinolysis ( 4.7 vs. $0 \%$; $p=0.24)$, and the PEITHO trial yielded similar findings (2.0 vs. $0.2 \% ; p=$ not reported) [30, 33••]. Interestingly, there were no hemorrhagic strokes in either treatment group in MAPPET-3 [32•]. Several factors may be associated with increased risk of intracranial hemorrhage. One retrospective analysis that utilized a national database of $49,500 \mathrm{PE}$ 
Table 4 Major and relative contraindications to fibrinolytics [3••]

\begin{tabular}{ll}
\hline Major contraindications & Relative contraindications \\
\hline Structural intracranial disease & $\mathrm{SBP}>180 \mathrm{mmHg}$ \\
Previous intracranial hemorrhage & $\mathrm{DBP}>110 \mathrm{mmHg}$ \\
Ischemic stroke within 3 months & $\begin{array}{c}\text { Recent bleeding } \\
\text { (nonintracranial) }\end{array}$ \\
Active bleeding & $\begin{array}{c}\text { Recent surgery or invasive } \\
\text { procedure }\end{array}$ \\
Recent brain or spinal surgery & Ischemic stroke more than \\
& 3 months previously \\
Recent head trauma with fracture & Anticoagulation \\
or brain injury & \\
Bleeding diathesis & Traumatic cardiopulmonary \\
& resuscitation \\
& Pericarditis or pericardial fluid \\
& Diabetic retinopathy \\
& Pregnancy \\
& Age $>75$ years \\
& Low body weight (e.g., $<60 \mathrm{~kg}$ ) \\
& Female sex \\
African-American race
\end{tabular}

$D B P$ diastolic blood pressure, $S B P$ systolic blood pressure

patients treated with fibrinolysis found a higher rate of intracerebral hemorrhage in patients age $>65$ years or with kidney disease compared to those age $\leq 65$ years without kidney disease (1.4 vs. $0.5 \% ; p<0.0001$ ) [36]. The prevalence of intracerebral hemorrhage was also increased in patients for whom PE was a secondary diagnosis compared to those in whom it was a primary diagnosis (1.7 vs. $0.6 \%$; $p<0.0001$ ) [36]. An elevated diastolic blood pressure has additionally been linked to excess intracranial bleed risk [37]. The incidence of fatal bleeding with fibrinolytics in hemodynamically stable PE patients with RVD appears to be low $(<1.0 \%)$ based on findings from MAPPET-3 and PEITHO and no different than when treated with anticoagulation alone $[32 \cdot 33 \bullet \cdot]$.

The available data consistently hint at increased risk of minor bleedings with adjunctive fibrinolytics in normotensive PE patients with RVD. In TIPES, minor bleedings occurred more frequently (14-fold) with fibrinolysis compared to placebo at 30 days ( $p=$ not reported) [28]. Fasullo et al. presented similar findings, with fibrinolytic use associated with a 2.6- and 2-fold excess risk of in-hospital $(p=0.20)$ and 6 months' minor bleedings (43.2 vs. $22 \%$; $p=0.005)$, respectively [29].

\section{Who Should Be Considered for Fibrinolytics?}

Given the lack of established benefit on early mortality and recurrent $\mathrm{PE}$ and increased risk of serious bleeding complications, caution is warranted when deciding whether to pursue this course of therapy in a particular patient with submassive PE. A review of current guideline recommendations as well as patient-specific characteristics may facilitate the decision process.

The most recent ACCP guidelines state that most patients who do not meet criteria for massive PE should not receive fibrinolytic therapy (grade 1C), with the exception of a select group of normotensive patients who are characterized as having a high risk of death or hypotension (i.e., submassive PE) but a low risk of bleeding (Grade 2C) [3*0]. The guidelines further outline that radiographic imaging and laboratory measures, in combination with evidence of clinical instability, may be used to identify this population. Radiographic and laboratory evidence includes RVD on electrocardiogram, echocardiography, or computed tomography, as well as biomarkers, such as elevated troponin and BNP; furthermore, clinical instability markers include a decrease in systolic blood pressure that is still greater than $90 \mathrm{mmHg}$, tachycardia, elevated jugular venous pressure, poor tissue perfusion, and hypoxemia [3・0]. Similarly, AHA proposed a treatment algorithm for use of fibrinolytics in submassive PE based upon analogous evidence of RVD and risk for hemodynamic instability [4••]. Although both recommending bodies stress the need to assess for PE severity based on RV function and evidence of clinical instability as well as to evaluate the risk of bleeding, there remains a lack of data to determine for which specific normotensive patients fibrinolysis is not only most effective but also reasonably safe.

In addition to the recommendations set forth by ACCP and AHA, current evidence suggests that several characteristics may be useful when evaluating whether fibrinolytics will be effective for submassive PE. These include patient age, extent of PE, and duration of symptoms. In PEITHO, patients $\leq 75$ years old who received fibrinolysis had a significantly reduced risk of the primary end point of all-cause death or hemodynamic collapse within 7 days (OR 0.33; CI 0.12-0.85), while those $>75$ years old did not derive the same benefit (OR 0.63; CI 0.23-1.66) [33••]. Parker et al. [38] determined that more extensive pulmonary perfusion defects prior to fibrinolytic therapy were correlated with more significant improvements in pulmonary function following fibrinolysis. This study, in addition to that of Daniels et al., confirmed there is an inverse relationship between duration of PE symptoms and improvement in lung reperfusion post-fibrinolysis; therefore, patients are likely to derive the most benefit from fibrinolytics if they are initiated soon after symptom onset $[38,39]$. The latter study, however, also determined that benefits from fibrinolysis may be observed if initiated within 14 days after symptom onset [39]. 
Consideration of a patient's risk of bleeding with fibrinolytic therapy must be assessed prior to initiation (see section on "Complications of Fibrinolysis" and Table 4).

\section{Fibrinolytic Choices}

Currently, the only two agents available on the US market that have been approved by the Food and Drug Administration (FDA) for use in patients with acute PE are urokinase and alteplase. Despite having an FDA indication for treating acute PE, streptokinase is no longer available for use. Both urokinase and alteplase only have indications for treating acute massive or hemodynamically unstable PE. Drug-specific characteristics may be considered when selecting an agent for fibrinolysis. Newer generation plasminogen activators have enhanced fibrin specificity to allow preferential activation of clot-bound plasminogen and limitation of systemic side effects. Unfortunately, since there are no definitive studies that have proved the superiority of one regimen over another, cost and hospital formulary availability might be the deciding factors on which agent is available for administration. Of note, the fibrinolytic agent evaluated in MAPPET-3 and Fasullo et al. was alteplase, whereas tenecteplase was studied in TIPES and PEITHO [28, 29, 32•, 33••]. See Table 2 for a summary of the pharmacokinetic and pharmacodynamic properties with fibrinolytics.

\section{Adjunctive Anticoagulation}

According to both AHA and ACCP, patients with confirmed $\mathrm{PE}$ and without contraindications should be initiated on therapeutic anticoagulation regardless of whether fibrinolytics are to be utilized $[3 \bullet \bullet, 4 \bullet \bullet$. Heparin anticoagulation is an option and includes intravenous or subcutaneous unfractionated heparin (UFH) targeted to an activated partial thromboplastin time (aPTT) of 2- to 2.5-times the control. Other options include subcutaneous low-molecularweight heparin and subcutaneous fondaparinux, a synthetic pentasaccharide $[3 \bullet \bullet, 4 \bullet \cdot]$. For patients with a history of heparin-induced thrombocytopenia, alternative anticoagulants, such as argatroban, lepirudin, or bivalirudin, should

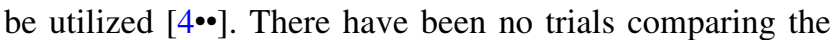
efficacy and safety of various anticoagulation regimens in conjunction with fibrinolysis, but in general most experience lies with intravenous UFH.

Practices regarding the concomitant administration of anticoagulants with fibrinolytics differ amongst countries. In the US, it is recommended to suspend intravenous UFH infusions during fibrinolytic administration [3••]. This cessation of anticoagulation, however, is not routinely performed in other countries, perhaps due to the differing administration requirements of the various fibrinolytics utilized; no studies have compared the two schemes [3••]. It is nevertheless recommended to recheck the aPTT immediately following the fibrinolytic infusion to ensure it is not at supratherapeutic levels prior to restarting the intravenous UFH infusion [3••].

Long-term anticoagulation with either a vitamin $\mathrm{K}$ antagonist (VKA) or an oral target-specific factor $\mathrm{Xa}$ inhibitor should commence as recommended for PE by the ACCP guidelines [3••]. If a VKA is selected, heparin or a heparin-alternative anticoagulant should continue for at least 5 days and until the international normalized ratio (INR) is therapeutic (2.0-3.0) for at least $24 \mathrm{~h}$ [3••]. Caution is warranted if heparin alternatives are utilized given the INR can be elevated in the presence of certain agents; therefore, modifications in INR monitoring and VKA dosage adjustments are recommended [40]. For the latter option, rivaroxaban is currently the only FDA-approved oral target-specific anticoagulant for prevention of recurrent $\mathrm{PE}$, and conversion to this therapy from parenteral anticoagulants is agent-specific [41]. It is important to note, however, that fibrinolysis-treated patients were excluded from the pivotal trial that investigated rivaroxaban use for acute PE [42]. Duration of long-term anticoagulation for $\mathrm{PE}$ is dependent upon whether the event is the first occurrence, whether a probable cause can be identified, and also the patient's bleeding risk; nonetheless, treatment should generally continue for a minimum of 3 months $[3 \bullet \bullet]$.

\section{Conclusion}

The role of adjunctive fibrinolysis in patients with submassive $\mathrm{PE}$ is controversial. Consistent evidence has demonstrated that the presence of RVD and/or myocardial necrosis identifies a subgroup of normotensive PE patients at elevated risk for early mortality and adverse outcomes. It has, therefore, been hoped that routine use of fibrinolytics may improve survival and prevent recurrent $\mathrm{PE}$ in this patient population given its established benefit in massive PE. The published and preliminary findings from the MAPPET-3 and PEITHO trials, respectively, have proven otherwise and revealed similar rates of both outcomes between treatment groups; this is not too surprising given the poor positive predictive values of RVD and/or myocardial necrosis for estimating mortality. The co-administration of fibrinolysis did yield a favorable effect on inhospital clinical deterioration and possible benefit on quality of life. However, given the potential for serious hemorrhagic complications associated with fibrinolytic therapy, its regular use in the broad range of submassive PE 
patients who have various levels of bleeding risks cannot be recommended.

The MAPPET-3 and PEITHO trials both enrolled patients with broad severities of risk. It is quite possible that adjunctive fibrinolysis may confer survival advantages and prevent PE recurrence in the severest subset of submassive PE patients. Current literature has not been able to clearly characterize these individuals. However, the presented findings of PEITHO are only preliminary, and its final results may shed some light onto this question. Future studies should attempt to identify and explore the use of fibrinolytics in this higher-risk subgroup.

In summary, the decision to administer adjunctive fibrinolytics in patients with submassive PE should be made on an individual basis. Particularly, it should be considered in those with more severe PE-related clinical manifestations, as outlined by ACCP and AHA, and a low risk of bleeding to help reduce the risk of in-hospital clinical deterioration and treatment escalation. Since there is currently no validated tool for predicting bleeding risk with fibrinolytics in acute PE, careful attention should be paid to the presence of comorbidities, such as kidney disease, and contraindications.

\section{Compliance with Ethics Guidelines}

Conflict of Interest Rathasen Prom, Ryan Dull, Stephanie Z. Kujawski, Iwona Rybak, and John Fanikos declare that they have no conflict of interest.

Human and Animal Rights and Informed Consent This article does not contain any studies with human or animal subjects performed by any of the authors.

\section{References}

Papers of particular interest, published recently, have been highlighted as:

- Of importance

-• Of major importance

1. Beckman MG, Hooper WC, Critchley SE, et al. Venous thromboembolism: a public health concern. Am J Prev Med. 2010;38:S495-501.

2. Stein PD, Beemath A, Matta F, et al. Clinical characteristics of patients with acute pulmonary embolism: data from PIOPED II. Am J Med. 2007;120:871-9.

3. • Kearon C, Akl EA, Comerota AJ, et al. Antithrombotic therapy for VTE disease: antithrombotic therapy and prevention of thrombosis, 9th ed: American College of Chest Physicians Evidence-Based Clinical Practice Guidelines. Chest. 2012;141: e419S-94S. This article provides guidelines on the use of antithrombotic therapy for the treatment and prevention of venous thromboembolism. In the section on acute pulmonary embolism, recommendations regarding the use of systemic fibrinolytics are provided.

4. • Jaff MR, McMurtry MS, Archer SL, et al. Management of massive and submassive pulmonary embolism, iliofemoral deep vein thrombosis, and chronic thromboembolic pulmonary hypertension: a scientific statement from the American Heart Association. Circulation. 2011;123:1788-1830. This consensus statement from the American Heart Association reviews the management strategies for massive and submassive pulmonary embolism, iliofemoral deep vein thrombosis, and chronic thromboembolic pulmonary hypertension. In patients with submassive PE, it reviews the efficacy and safety outcomes associated with systemic fibrinolsis and provides recommendations on the use of systemic fibrinolysis.

5. Goldhaber SZ, Visani L, De Rosa M. Acute pulmonary embolism: clinical outcomes in the international cooperative pulmonary embolism registry (ICOPER). Lancet. 1999;353:1386-9.

6. Kasper W, Konstantinides S, Geibel A, et al. Management strategies and determinants of outcome in acute major pulmonary embolism: results of a multicenter registry. J Am Coll Cardiol. 1997;30:1165-71.

7. Binder L, Pieske B, Olschewski M, et al. N-terminal pro-brain natriuretic peptide or troponin testing followed by echocardiography for risk stratification of acute pulmonary embolism. Circulation. 2005;112:1573-9.

8. Piazza G. Submassive pulmonary embolism. JAMA. 2013;309: $171-80$.

9. Grifoni S, Olivotto I, Cecchini P, et al. Short-term clinical outcome of patients with acute pulmonary embolism, normal blood pressure, and echocardiographic right ventricular dysfunction. Circulation. 2000;101:2817-22.

10. Konstantinides S, Geibel A, Olschewski M, et al. Association between thrombolytic treatment and the prognosis of hemodynamically stable patients with major pulmonary embolism: results of a multicenter registry. Circulation. 1997;96:882-8.

11. Kucher N, Rossi E, De Rosa M, et al. Prognostic role of echocardiography among patients with acute pulmonary embolism and a systolic arterial pressure of $90 \mathrm{~mm} \mathrm{Hg}$ or higher. Arch Intern Med. 2005;165:1777-81.

12. Ten Wolde M, Sohne M, Quak E, et al. Prognostic value of echocardiographically assessed right ventricular dysfunction in patients with pulmonary embolism. Arch Intern Med. 2004;164: 1685-9.

13. Sanchez O, Trinquart L, Colombet I, et al. Prognostic value of right ventricular dysfunction in patients with haemodynamically stable pulmonary embolism: a systematic review. Eur Heart J. 2008;29:1569-77.

14. Jimenez D, Aujesky D, Moores L, et al. Combinations of prognostic tools for identification of high-risk normotensive patients with acute symptomatic pulmonary embolism. Thorax. 2011;66: 75-81.

15. Bova C, Pesavento R, Marchiori A, et al. Risk stratification and outcomes in hemodynamically stable patients with acute pulmonary embolism: a prospective, multicentre, cohort study with three months of follow-up. J Thromb Haemost. 2009;7:938-44.

16. Stein PD, Beemath A, Matta F, et al. Enlarged right ventricle without shock in acute pulmonary embolism: prognosis. Am J Med. 2008;121:34-42.

17. Klok FA, Mos IC, Huisman MV. Brain-type natriuretic peptide levels in the prediction of adverse outcome in patients with pulmonary embolism: a systematic review and meta-analysis. Am J Respir Crit Care Med. 2008;178:425-30.

18. Pruszczyk P, Bochowicz A, Torbicki A, et al. Cardiac troponin T monitoring identifies high-risk group of normotensive patients with acute pulmonary embolism. Chest. 2003;123:1947-52. 
19. Becattini C, Vedovati MC, Agnelli G. Prognostic value of troponins in acute pulmonary embolism: a meta-analysis. Circulation. 2007;116:427-33.

20. Scridon T, Scridon C, Skali H, et al. Prognostic significance of troponin elevation and right ventricular enlargement in acute pulmonary embolism. Am J Cardiol. 2005;96:303-5.

21. Haire WD. Pharmacology of fibrinolysis. Chest. 1992;101:91S$7 \mathrm{~S}$.

22. Product Information. Retavase (reteplase). Bedminster: EKR Therapeutics, Inc.; 2009.

23. Product Monograph. Streptase (streptokinase). Ottawa: CSL Behring Canada, Inc.; 2007.

24. Product Information. Kinlytic (urokinase). Tucson: ImaRx Therapeutics, Inc.; 2007.

25. Verstraete M. Third-generation thrombolytic drugs. Am J Med. 2000;109:52-8.

26. Product Information. Activase (alteplase). San Francisco: Genentech, Inc.; 2011

27. Information Product. TNKase (tenecteplase). San Francisco: Genentech, Inc.; 2011.

28. Becattini C, Agnelli G, Salvi A, et al. Bolus tenecteplase for right ventricle dysfunction in hemodynamically stable patients with pulmonary embolism. Thromb Res. 2010;125:e82-6.

29. Fasullo S, Scalzo S, Maringhini G, et al. Six-month echocardiographic study in patients with submassive pulmonary embolism and right ventricle dysfunction: comparison of thrombolysis with heparin. Am J Med Sci. 2011;341:33-9.

30. Hamel E, Pacouret G, Vincentelli D, et al. Thrombolysis or heparin therapy in massive pulmonary embolism with right ventricular dilation: results from a 128-patient monocenter registry. Chest. 2001;120:120-5.

31. Goldhaber SZ, Haire WD, Feldstein ML, et al. Alteplase versus heparin in acute pulmonary embolism: randomised trial assessing right-ventricular function and pulmonary perfusion. Lancet. 1993;341:507-11.

32. - Konstantinides S, Geibel A, Heusel G, et al. Heparin plus alteplase compared with heparin alone in patients with submassive pulmonary embolism. N Engl J Med. 2002;347:1143-1150. This is the largest published trial to date comparing heparin alone to fibrinolysis (alteplase) plus heparin. There was no difference in mortality, but patients receiving heparin alone had a higher incidence of clinical deterioration requiring treatment escalation (catecholamine infusion, secondary thrombolysis, endotracheal intubation, cardiopulmonary resuscitation, emergency surgical embolectomy, or thrombus catheter fragmentation).

33. •- Pulmonary embolism thrombolysis study. http://clinical trialresults.org/Slides/ACC\%202013/Konstantinides_PEITHO_ ACC\%202013.pdf (accessed 2013 July 16). This is the largest trial (not yet published) to date comparing heparin alone to fibrinolysis (tenecteplase) plus heparin. Like MAPPET-3, there was no difference in mortality; however, patients receiving heparin alone had a higher incidence of hemodynamic collapse during hospitalization.

34. Tardy B, Venet C, Zeni F, et al. Short term effect of recombinant tissue plasminogen activator in patients with hemodynamically stable acute pulmonary embolism: results of a meta-analysis involving 464 patients. Thromb Res. 2009;124:672-7.

35. Kline JA, Steuerwald MT, Marchick MR, et al. Prospective evaluation of right ventricular function and functional status 6 months after acute submassive pulmonary embolism: frequency of persistent or subsequent elevation in estimated pulmonary artery pressure. Chest. 2009;136:1202-10.

36. Stein PD, Matta F, Steinberger DS, et al. Intracerebral hemorrhage with thrombolytic therapy for acute pulmonary embolism. Am J Med. 2012;125:50-6.

37. Kanter DS, Mikkola KM, Patel SR, et al. Thrombolytic therapy for pulmonary embolism. Frequency of intracranial hemorrhage and associated risk factors. Chest. 1997;111:1241-5.

38. Parker JA, Drum DE, Feldstein ML, et al. Lung scan evaluation of thrombolytic therapy for pulmonary embolism. J Nucl Med. 1995;36:364-8.

39. Daniels LB, Parker JA, Patel SR, et al. Relation of duration of symptoms with response to thrombolytic therapy in pulmonary embolism. Am J Cardiol. 1997;80:184-8.

40. Information Product. Argatroban. Research triangle park. Durham: GlaxoSmithKline, Inc.; 2012.

41. Information Product. Xarelto (rivaroxaban). Titusville: Janssen Pharmaceuticals, Inc.; 2012.

42. Buller HR, Prins MH, Lensin AW, et al. Oral rivaroxaban for the treatment of symptomatic pulmonary embolism. N Engl J Med. 2012;366:1287-97. 